\title{
Bezoar gástrico de plástico como causa de pancreatitis aguda: reporte de un caso y revisión de la literatura
}

\author{
Audel Pedroza ${ }^{1}$, Fernando Aguirre ${ }^{1}$, Gina Parra ${ }^{1}$, Ricardo Buitrago ${ }^{1}$, Anwar Medellín ${ }^{1}$, Carlos López ${ }^{1}$, \\ Hugo Cómbita ${ }^{1}$, Álvaro Silva ${ }^{1}$, Catalina Riaño ${ }^{1}$, Sebastián Martínez ${ }^{2}$, Rafael Santos ${ }^{2}$, Eliana Cortés ${ }^{2}$, \\ INGRID RIVERA ${ }^{2}$, Luis Felipe CABRERA ${ }^{2}$
}

Palabras clave: estómago; migración de cuerpo extraño; pancreatitis; diagnóstico diferencial; terapéutica; laparoscopía.

\section{Resumen}

Introducción. Un bezoar se define como un elemento no digerible atrapado en algún punto del tubo digestivo, el cual puede ser ingerido de forma intencional o accidental. El primer reporte de un bezoar en la literatura médica lo hizo Baudamant en 1779.

Caso clínico. Se presenta un caso de un bezoar de plástico, un cepillo dental, como causa de pancreatitis aguda y su manejo por laparoscopia, con una revisión de la literatura.

Discusión y conclusiones. Aunque los bezoares gástricos son poco frecuentes, y más aún el de plástico, es importante tenerlos en cuenta como causa de pancreatitis aguda. Definitivamente, el abordaje laparoscópico es el ideal en pacientes sin obstrucción intestinal o inestabilidad hemodinámica.

1 Cirugía General, Hospital Simón Bolívar E.S.E., Bogotá, D.C., Colombia

2 Residente de Cirugía General, Hospital Simón Bolívar E.S.E., Universidad El Bosque, Bogotá, D.C., Colombia

Fecha de recibido: 6 de julio de 2016

Fecha de aprobación: 22 de julio de 2016

Citar como: Pedroza A, Aguirre F, Parra G, Buitrago R, Medellín A, López $\mathrm{C}$, et al. Bezoar gástrico de plástico como causa de pancreatitis aguda: reporte de un caso y revisión de la literatura. Rev Colomb Cir. 2017;32:152-56.

\section{Introducción}

Un bezoar se define como un elemento no digerible atrapado en algún punto del tubo digestivo, el cual puede ser ingerido de forma intencional o accidental. El primer reporte de un bezoar en la literatura médica lo hizo Baudamant en $1779^{1}$, como un hallazgo incidental durante una autopsia.

Se pueden clasificar según el elemento que los conforma y, en general, existen: fitobezoar (vegetales y frutas), tricobezoar (cabello), onicobezoar (uñas), farmacobezoar (medicamentos), lactobezoar (proteína de la leche) y bezoar de plástico, entre otros. Se pueden ubicar en cualquier sitio del tubo digestivo, aunque el estómago es la ubicación más frecuente. Por lo general, al hacerse el diagnóstico de un bezoar este debe ser retirado o disuelto, ya que puede causar obstrucción gástrica o intestinal, íleo, úlceras, sangrado y, con menor frecuencia, pancreatitis aguda ${ }^{2,3}$.

En el presente trabajo, nos permitimos presentar un caso de un bezoar de plástico como causa de pancreatitis aguda y su manejo por laparoscopia, con una revisión exhaustiva de la literatura ${ }^{1-3}$.

\section{Caso clínico}

Se trata de una mujer de 39 años de edad, con un cuadro clínico de un día de evolución consistente en astenia, adinamia, dolor abdominal en el mesogastrio que se 
irradiaba en banda, de intensidad 8/10, y múltiples episodios eméticos. Tenía el antecedente de dos episodios de pancreatitis aguda leve sin etiología establecida y colecistectomía laparoscópica un año antes. No refirió otros síntomas en la revisión por sistemas.

En el examen físico, presentaba dolor en el mesogastrio, sin signos de irritación peritoneal ni masas. En los exámenes de laboratorio se encontró amilasa sérica de $872 \mathrm{U} / \mathrm{L}$, fosfatasa alcalina de $277 \mathrm{U} / \mathrm{L}$, perfil hepático normal y, en el hemograma, leucocitosis de 13.540 por $\mathrm{mm}^{3}$.

Se consideró una pancreatitis aguda leve en curso, de origen por establecer, con puntuación de 1 según la escala de Marshall, y de 5 según la Acute Physiology and Chronic Health Evaluation (APACHE II). Con el fin de descartar una coledocolitiasis residual como causa del cuadro clínico, se practicó una ultrasonografía biliopancreática endoscópica, en la cual se observó un colédoco de $3 \mathrm{~mm}$ de diámetro sin cálculos, edema del páncreas y un cuerpo extraño que correspondía a un cepillo dental con cerdas compactadas, en la primera y la segunda porción duodenal, con áreas de grave erosión (figura 1). Se intentó su retiro endoscópico, pero se encontró una importante resistencia al paso por el cardias, por lo cual se suspendió el procedimiento debido al riesgo de perforación.

Se decidió practicar una extracción quirúrgica bajo anestesia general, por vía laparoscópica. El procedimiento se llevó a cabo sin complicaciones, con una técnica de cuatro puertos en posición francesa, a través de una gastrotomía anterior a nivel antrocorporal, empleando un disector ultrasónico de tipo Harmonic ${ }^{\circledR}$ (Ethicon). Se identificó el bezoar de plástico, se extrajo con pinzas laparoscópicas de agarre y se introdujo en una endobolsa para su posterior extracción de la cavidad abdominal (figura 2). La gastrotomía se cerró con sutura laparoscópica y nudos intracorpóreos, con puntos continuos simples de poliglactina 3-0 vicril ${ }^{\circledR}$ (Ethicon) en un solo plano. No se hizo la prueba de azul de metileno para fugas y no se requirió endoscopia intraoperatoria (figura 3). La duración de la cirugía fue de una hora y media, y hubo $10 \mathrm{ml}$ de sangrado.

La paciente presentó una adecuada evolución posoperatoria y fue dada de alta al tercer día.

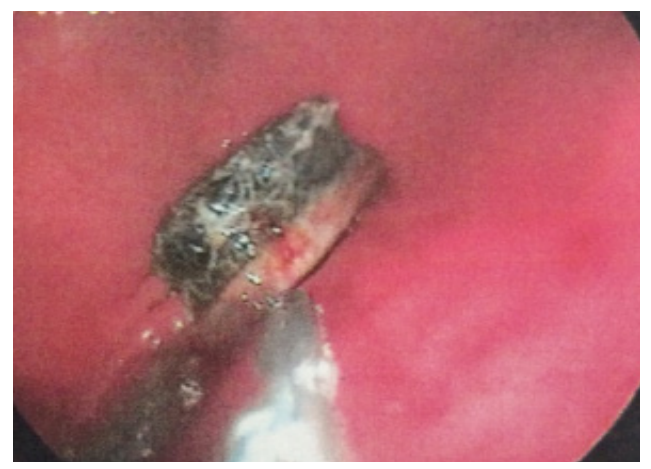

FiguRA 1. Bezoar gástrico de plástico, un cepillo dental

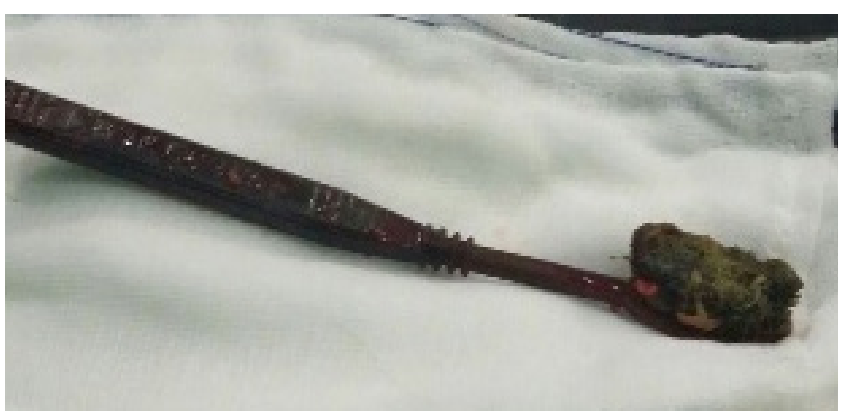

Figura 2. Bezoar de plástico (cepillo dental) extraído de la cavidad abdominal por vía laparoscópica

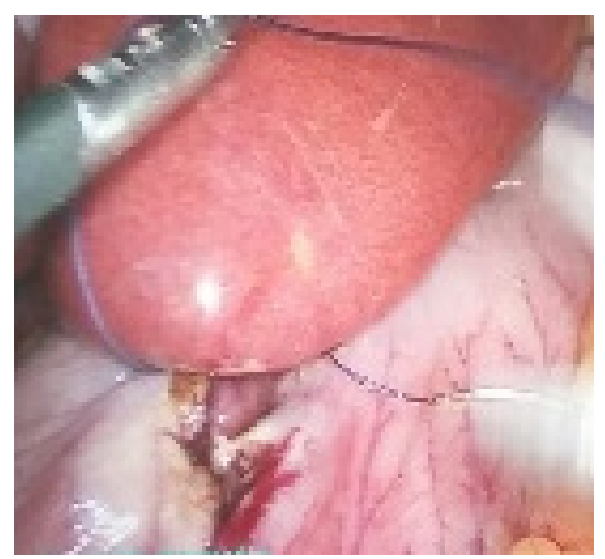

FIGURA 3. Cierre de gastrotomía anterior por sutura laparoscópica y nudos intracorpóreos

\section{Discusión}

La palabra bezoar proviene del arábigo bāzahr, que significa antídoto; los bezoares extraídos de los animales sacrificados fueron empleados como pociones hasta el siglo XIX. 
Los bezoares del tubo digestivo, se consideran poco frecuentes, con una incidencia que ha venido en disminución con el paso del tiempo; Ahn, et al., reportaron una de $0,43 \%$ durante un periodo de siete años, y Mihai, et al., una de $0,068 \%$ durante un periodo de 20 años ${ }^{4,5}$. Se presentan con mayor frecuencia en pacientes de sexo femenino con trastornos psiquiátricos, como en el presente caso; sin embargo, su prevalencia varía según las poblaciones y sus costumbres, como en Corea y Japón, en donde predominan los fitobezoares ${ }^{6,7}$.

Los bezoares se clasifican según su composición, como lo mencionamos anteriormente. En este caso, el elemento que formaba el bezoar era de plástico (cepillo dental), el cual se considera un material muy infrecuente y solo se presenta en el $0,1 \%$ de los casos, ya que predominan los fitobezoares y los tricobezoares ${ }^{8}$

Se cree que la mayoría de los bezoares se originan por una alteración en el vaciamiento gástrico, y existen factores predisponentes, como cirugía gástrica previa (por ejemplo, gastrectomía subtotal), vagotomía mas piloroplastia, enfermedad úlcero-péptica, enfermedad de Crohn, cáncer gástrico, diabetes mellitus, tercera edad, deshidratación e hipotiroidismo. Estas condiciones llevan a disminución del $\mathrm{pH}$ gástrico, estasis gástrica y pérdida de la motilidad gástrica o estenosis pilórica ${ }^{9}$. En el presente caso, tras una ingestión accidental del cepillo dental, se formó el bezoar pues, por su gran tamaño no migró y por ser material plástico no se digirió en el estómago, ya que la paciente no presentaba ninguno de estos factores de riesgo ${ }^{8,9}$.

Esta enfermedad se puede presentar de forma asintomática y ser un hallazgo incidental durante una endoscopia de vías digestivas altas, hasta en $0,5 \%$ de los casos, o manifestarse con hemorragia de las vías digestivas altas, en 41,2 a 64,5\%, como lo reportaron Lee, et al., ${ }^{10}$ seguida de obstrucción intestinal. Además, se pueden presentar otras complicaciones menos frecuentes, como perforación y peritonitis, enteropatía perdedora de proteína, apendicitis aguda o, como en este caso, pancreatitis aguda. En este, el bezoar de plástico iba desde la curvatura menor del cuerpo gástrico, por la primera y hasta la segunda porción del duodeno; simulaba un síndrome de Rapunzel y generaba comprensión proximal y distal de la ampolla de Váter, obstruyendo el conducto de Wirsung y, por tanto, el drenaje de la secreción pancreática ${ }^{11}$.

El tratamiento estándar consiste en la disolución química o la extracción mecánica del bezoar. Se pueden emplear infusiones o bolos de Coca-Cola ${ }^{\circledR}, 3.000$ $\mathrm{ml}$ o $100 \mathrm{ml}$ cada 15 minutos durante 12 horas -como lo reportaron Chung, et al. ${ }^{12}$, con una eficacia de 42 a $91,2 \%-$, papaína, la cual no ha demostrado ser mejor que el agua, y celulosa, la cual está descontinuada y solo se encuentra en combinación con otros suplementos dietéticos y no ha demostrado una adecuada eficacia ${ }^{10,12}$.

Para su extracción o fragmentación, se puede emplear la vía endoscópica, usando diferentes dispositivos, como pinzas de biopsia, pinzas cocodrilo, pinzas para polipectomía, canastillas, coagulador de argón plasma, litotriptor, bisturí endoscópico electroquirúrgico o, como lo reportaron Kurt, et al., un polifilamento oval endoscópico diseñado específicamente para el manejo de bezoares ${ }^{12,13}$. Sin embargo, estos procedimientos solo son efectivos con los fitobezoares; debido a su alta densidad, el tricobezoar y el bezoar de plástico son resistentes a la fragmentación y la degradación enzimática, como lo evidenciaron Frohna, et al., con una tasa de éxito de solo $0,5 \%{ }^{14}$.

Cuando los anteriores tratamientos médicos o endoscópicos fallan, se requiere la extracción quirúrgica del bezoar. Esta se puede llevar a cabo por vía abierta o laparoscópica, según el estado clínico del paciente y el tamaño del bezoar, como lo informaron Diefenbach, et al., en un estudio retrospectivo y descriptivo de siete pacientes, de los cuales cinco requirieron laparotomía $15,16,17$.

La primera extracción laparoscópica de un bezoar gástrico la realizó Nirasawa en 1998. En un estudio retrospectivo, Yau, et al. ${ }^{16}$, demostraron que el abordaje laparoscópico presenta una menor tasa de complicaciones locales, menor dolor posoperatorio, menos infecciones del sitio operatorio, un tiempo quirúrgico similar, un resultado estético favorable y menos tiempo de hospitalización ${ }^{17,18}$. Dorn, et al., describieron una técnica por vía laparoscópica, en la cual se coloca un puerto intragástrico para remover en pedazos el bezoar, con el fin de minimizar el riesgo de contaminación de la cavidad peritoneal; sin embargo, el tiempo quirúrgico se prolongó hasta en seis horas ${ }^{18}$. 
El abordaje llevado a cabo en la presente paciente se basó en la técnica descrita por Kohler, et al. ${ }^{19}$, en la cual se practica una gastrotomía anterior y el bezoar se extrae en una endobolsa; aunque con esta es más frecuente la contaminación de la cavidad abdominal, en nuestro caso no se produjeron colecciones posoperatorias, el tiempo quirúrgico fue corto y el resultado estético fue aceptable ${ }^{17,18,19}$.

Se han descrito otros tratamientos para los bezoares, como los fármacos que aumentan la motilidad gastrointestinal (procinéticos como itoprida, mosaprida y metoclopramida), para que se pueda romper el bezoar y expulsarlo. Por otra parte, en pacientes asintomáticos, se puede hacer seguimiento y esperar la expulsión espontánea. Estos no eran útiles en el presente caso, por el tamaño y la consistencia del bezoar, y por la sintomatología de la paciente $20,21,22$.

\section{Conclusiones}

Aunque los bezoares gástricos son poco frecuentes y, más aún, el bezoar de plástico, es importante tenerlos en cuenta como una causa de pancreatitis aguda. Definitivamente, el abordaje laparoscópico es el ideal en pacientes sin obstrucción intestinal o inestabilidad hemodinámica. La anamnesis completa nos permite determinar con mayor prontitud el diagnóstico.

\section{Financiación}

Ninguna

\section{Conflicto de intereses}

Todos nosotros declaramos no tener ningún conflicto de intereses y que no tenemos relación económica alguna con una entidad comercial

\title{
Gastric plastic bezoar causing acute pancreatitis: case report and literature review
}

\begin{abstract}
Introduction: A bezoar is defined as an indigestible element trapped somewhere in the gastrointestinal tract, which can be ingested intentionally or accidentally. The first report of a Bezoar in the medical literature was by $W$. Baudamant in 1779.

Case report: We present the case of a plastic bezoar, a toothbrush, as the cause of acute pancreatitis and its management by laparoscopy, and a literature review.

Discussion and conclusions: Although gastric bezoars are rare conditions, and furthermore so plastic bezoars, it is important to take them into account as a rare cause of acute pancreatitis. Definitely the laparoscopic approach is ideal for patients without intestinal obstruction or hemodynamic instability.
\end{abstract}

Key words: Stomach; foreign-body migration; pancreatitis; diagnosis, differential; therapeutics; laparoscopy.

\section{Referencias}

1. Baudamant W. Memoire sur des cheveux trouver dans l'estomac et dans les intestins grêles. Jour de Medecine, Chirurgie, Pharmacie. 1779;52:507-14.

2. Zhang RL, Yang ZL, Fan BG. Huge gastric disopyrobezoar: a case report and review of literatures. World J Gastroenterol 2008; 14: $152-154$

3. Iwamuro M, Okada H, Matsueda K, Inaba T, Kusumoto C, Imagawa $\mathrm{A}$, et al.Review of the diagnosis and management of gastrointestinal bezoars. World J Gastrointest Endosc. 2015;7:336-45.
4. Ahn YH, Maturu P, Steinheber FU, Goldman JM. Association of diabetes mellitus with gastric bezoar formation. Arch Intern Med. 1987;147:527-8.

5. Mihai C, Mihai B, Drug V, Cijevschi Prelipcean C. Gastric bezoars---diagnostic and therapeutic challenges. J Gastrointestin Liver Dis. 2013;22:111.

6. Altintoprak F, Degirmenci B, Dikicier E, Cakmak G, Kivilcim $\mathrm{T}$, Akbulut $\mathrm{G}$, et al. CT findings of patients with small bowel obstruction due to bezoar: a descriptive study. Scientific World J. 2013;2013:298392. 
7. Yeh J, Saul T, Gingrich A, Wassermann J. Bezoar. J Emerg Med. 2013;45:615-6.

8. Campos RR, Paricio PP, Albasini JLA, Riquelme Riquelme J, Cifuentes Tebar J, Luján Mompeán JA. Gastrointestinal bezoars. Presentation of 60 cases. Dig Surg. 1990;7:39-44.

9. Simsek Z, Altinbas A, Yuksel I, Yuksel O. Effective treatment with pineapple juice in small bowel obstruction due to phytobezoar in a gastrectomized patient. Dig Endosc. 2011;23:197.

10. Lee BJ, Park JJ, Chun HJ, Kim JH, Yeon JE, Jeen YT, et al. How good is cola for dissolution of gastric phytobezoars? World J Gastroenterol. 2009;15:2265-9.

11. Iwamuro M, Kawai Y, Shiraha H, Takaki A, Okada H, Yamamoto $\mathrm{K}$. In vitro analysis of gastric phytobezoar dissolubility by cocacola, coca-cola zero, cellulase, and papain. J Clin Gastroenterol. 2014;48: 190-191.

12. Chung YW, Han DS, Park YK, Son BK, Paik CH, Jeon YC, Sohn JH. Huge gastric diospyrobezoars successfully treated by oral intake and endoscopic injection of Coca-Cola. Dig Liver Dis 2006; 38 : $515-517$

13. Iwamuro M, Tanaka S, Shiode J, Imagawa A, Mizuno M, Fujiki S, et al. Clinical characteristics and treatment outcomes of nineteen Japanese patients with gastrointestinal bezoars. Intern Med. 2014;53:1099-105.

14. Kurt M, Posul E, Yilmaz B, Korkmaz U. Endoscopic removal of gastric bezoars: an easy technique. Gastrointest Endosc. 2014; 2014;80:895-6.

15. Frohna WJ. Metamucil bezoar: an unusual cause of small bowel obstruction. Am J Emerg Med. 1992;10:393-5.

16. Yau KK, Siu WT, Law BK, Cheung HY, Ha JP, Li MK. Laparoscopic approach compared with conventional open approach for bezoar-induced small-bowel obstruction. Arch Surg. 2005;140(10):972-975.

17. Kannan NL, Singaraju H, Sim SW. Laparoscopic-assisted removal of gastric trichobezoar: A novel technique to reduce operative complications and time. J Pediatr Surg. 2013;48:1826-7.

18. Diefenbach GJ, Reitman D, Williamson DA. Trichotillomania: A challenge to research and practice. Clin Psychol Rev. 2000;20:289-309.

19. Dorn H, Gillick J, Stringel G. Laparoscopic intragastric removal of giant trichobezoar. JSLS. 2010;14:259-62.

20. Kohler J. E., Millie M., Neuger E. Trichobezoar causing pancreatitis: first reported case of Rapunzel syndrome in a boy in North America. Journal of Pediatric Surgery. 2012;47(3):E17-E19.

21. Fraser J, Leys C, St Peter S. Laparoscopic removal of a gastric trichobezoar in a pediatric patient. J Laparoendosc Adv Surg Tech A. 2009;19:835-7.

22. Kadian RS, Rose JF, Mann NS. Gastric bezoars --spontaneous resolution. Am J Gastroenterol. 1978;70:79-82.

23. Ortiz-Soto J, Suárez-Nadal J, Nava-Carillo A. Tricotextilo bezoar, síndrome de Rapunzel. Presentación de un caso. Revista Mexicana de Cirugía Pediátrica. 2005;12:51-4.

$$
\begin{aligned}
& \text { Correspondencia: Audel Pedroza, MD } \\
& \text { Correo electrónico: audelpedroza@hotmail.com } \\
& \text { Bogotá, D.C., Colombia }
\end{aligned}
$$

